\title{
Neurogenic effects on the palatopharyngeal muscle in patients with obstructive sleep apnoea: a muscle biopsy study
}

\author{
Lars Edström, Håkan Larsson, Lars Larsson
}

\begin{abstract}
Muscle biopsies from the palatopharyngeal muscle of eight patients with obstructive sleep apnoea were performed during uvulopalatopharyngoplasty. Control biopsies were performed during tonsillectomy in seven control patients with no history of symptoms suggesting obstructive sleep apnoea. The diagnosis was based on the patient's history and a whole night recording of arterial oxygen saturation and respiration movements. The mean number of oxygen desaturations $\geqslant 4 \%$ per sleeping hour was 39 (range 7-80) in patients with obstructive sleep apnoea. In the control patients the occurrence of muscle fibre type and size relation between type I and type II fibres were comparable to what is found in the quadriceps femoris muscle, but the mean size of the fibres was $<25 \%$ of what is found in limb muscles.
\end{abstract}

All biopsies from patients with obstructive sleep apnoea showed abnormalities. Atrophy with a fascicular distribution, increased number of angulated atrophic fibres, a twin or multiple peak distribution of the fibre size spectra, and an abnormal distribution of fibre types in many muscle fascicles corresponding to "type grouping" all points to a neurogenic alteration. This neurogenic lesion may be a primary phenomenon or secondary to the trauma of repetitive and prolonged stretching of the pharyngeal structures during apnoeas. A disturbance of the function of the dilating muscles of the upper airway may be important in causing the abnormal airway collapse seen in obstructive sleep apnoea.

$(\mathcal{F}$ Neurol Neurosurg Psychiatry 1992;55:916-920)

Department of

Nars Edstrom

Department of

Clinical

Neurophysiology

Lars Larsson

Department of

Otorhinolaryngology, Karolinska Institute, South Hospital, Stockholm, Sweden Håkan Larsson

Correspondence to: Dr H Larsson, Öronkliniken, Södersjukhuset, S-118 83 Stockholm, Sweden Received 9 May 1991 and in final revised form 11 December 1991.

Accepted 30 January 1992 macromorphology of the uvular muscle in patients with obstructive sleep apnoea ${ }^{2}$ but there has been no structural evaluation at the cellular or subcellular level.

To determine whether histopathological changes were present in the pharyngeal muscles in patients with obstructive sleep apnoea, muscle biopsy specimens from the palatopharyngeal muscle were obtained in eight patients with obstructive sleep apnoea undergoing uvulopalatopharyngoplasty ${ }^{3}$ and in seven control patients undergoing tonsillectomy.

\section{Material and methods}

PATIENTS

Eight patients with obstructive sleep apnoea, all male and with a mean age of 43 (range 28-54) years, were examined. One patient had arterial hypertension and one had had a minor cerebral infarction but with no remaining symptoms. The diagnosis of obstructive sleep apnoea was based on the patient's history, clinical examination, and a whole night recording of arterial oxygen saturation (Biox 3700, earprobe) and of respiration and body movements by means of a static charge sensitive bed. ${ }^{4}$ This type of recording with diagnostic criteria has previously been described in detail. ${ }^{5}$ The oxygen desaturation index was calculated from the sleep apnoea recordings as the average number per sleeping hour, of oxygen desaturations $\geqslant 4 \%$ below baseline during periods with obstructive breathing pattern. The mean oxygen desaturation index for the eight patients with obstructive sleep apnoea was 39 (7-80). The mean nadir arterial oxygen saturation was $70 \%(47-85 \%)$. All patients had normal results on spirometry and normal arterial blood gas analyses during the daytime. Holter electrocardiography was performed in seven patients and all were normal. Thyroid stimulating hormone concentrations were normal in all patients. No neurological evaluation or investigations were undertaken in the patients.

The controls were seven men with a mean age of 39 (range 17-59) years and with no history of snoring or other symptoms suggesting obstructive sleep apnoea. Six were scheduled for tonsillectomy due to chronic tonsillitis, and in one the tonsillectomy was performed in a search for the primary tumour of a lymph node metastasis in the neck; no tumour was found in the tonsil or in the pharynx.

The patients with obstructive sleep apnoea were more obese than the controls. The mean 
body mass index (weight (kg)/(length $(\mathrm{m}))^{2}$ was $31.9(25.6-36.5)$ in the patients and 23.8 $(18 \cdot 7-28 \cdot 1)$ in the controls.

BIOPSY PROCEDURE

Tonsillectomy was performed as a part of the uvulopalatopharyngoplasty in all patients with obstructive sleep apnoea and as the only procedure in the control patients. After removal of the tonsils the palatopharyngeal muscle was identified and a piece from the cranial part of the muscle about $10 \times 5 \times 5 \mathrm{~mm}$ was.removed. The specimens were embedded in sterile cloths moistened with $0.9 \%$ saline and immediately transported to the laboratory.

MORPHOLOGICAL AND HISTOCHEMICAL TECHNIQUES Most of each specimen was freshly frozen in liquid freon-13, cooled by liquid nitrogen $\left(-190^{\circ} \mathrm{C}\right)$ and stored in a deep freezer at $-75^{\circ} \mathrm{C}$, and a part was fixed in $2.5 \%$ glutaraldehyde in phosphate buffer.

All specimens were examined with light microscopy. Freshly frozen material was cut in a cryostat at $-25^{\circ} \mathrm{C}$. Cryosections $(5-15 \mu \mathrm{m})$ were stained with hematoxylin-eosin and modified trichrome for adnosine triphophatase (ATPase) and NADH-TR. ${ }^{6}$

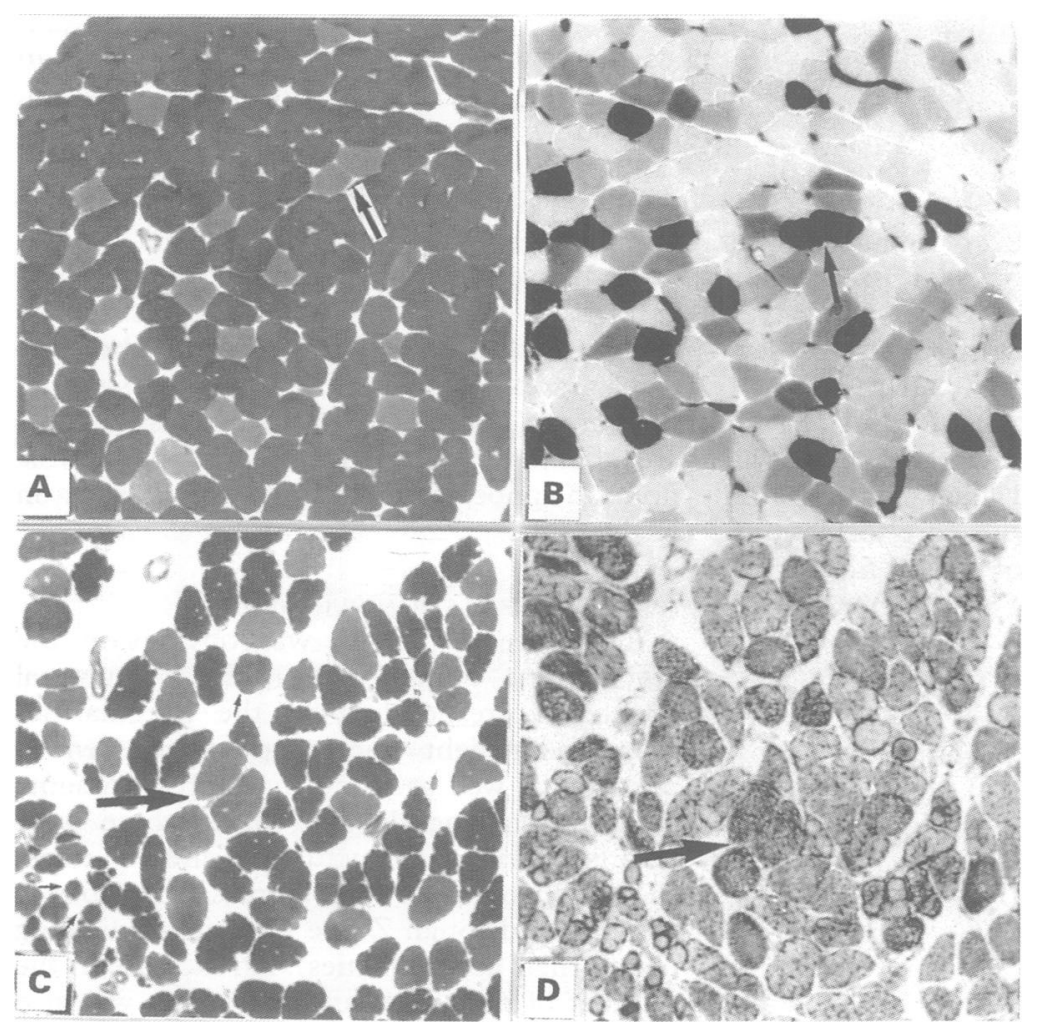

Figure 1 Serial cross sections of palatopharyngeal muscle from control patient ( $a, b)$ and patient with obstructive sleep apnoea $(c, d)$. Control specimen was stained for myosin-ATPase at $\mathrm{pH} 9.4$ (a) and after acid preincubation at $\mathrm{pH} 4.6$ (b). Arrow points to two type I fibres, lightly stained by preincubation at alkaline $p H$ and darkly stained at acid $\mathrm{pH}$. Fibres with different levels of staining in (b) correspond to subtypes of type II; the unstained fibres are type ILA and intermediate stained are type IIB.

The apnoea patient's specimen was stained for myosin-ATPase at pH 9.4 (c) and for $N A D H-T R$ (d). Note dispersion of fibre sizes in all sections and irregular muscle fibre structure (d). Thick arrow points to three typical type I fibres of large cross sectional area. These fibres are of normal structure in $b$ (NADH-TR) but surrounded by numerous fibres with irregular staining deposits. The distinction between type I and type II fibres at pH 9.4 (a) is not as clear cut as normal. Thin arrows point to some of the fibres with intermediate staining.
Muscle fibre type nomenclature was based on the ATPase content of fibres according to Brooke and Kaiser. ${ }^{7}$ Thus fibres with a high content of acid stable ATPase and a low content of alkali stable ATPase were termed type I, and fibres with the opposite staining pattern were termed type II. Subtypes of type II (A, B, and C) were also observed by using different $\mathrm{pH}$ levels $(4 \cdot 3$ and $4 \cdot 6)$ for acid preincubation.

Muscle fibre size- that is, the cross sectional area and the "lesser diameter", was measured directly from the microscope via a TV overlay with the aid of a digitiser connected to a computer (Videoplan, Konron Bildanalyse $\mathrm{GmBH}$, Munich). Measurements were made on 100 type I and 100 type II fibres or as many as possible in each biopsy (50-100 type I fibres and 100 type II fibres were measured in each patient). The form factor (fibre area $\times(\pi 4 \times$ maximum diameter $\times$ minimum diameter $)^{-1}$ ) was calculated for each fibre. The form factor for a circle and for an ellipse is 1 and for irregular structures $<1$.

For evaluation by transmission electron microscopy (TEM), specimens fixed in glutaraldehyde were washed in buffer, cut into blocks approximately $2 \times 0.5 \mathrm{~mm}$ and post fixed in osmium oxide in phosphate buffered saline, dehydrated in alcohol, and embedded in Epon. Semithin sections were stained in toluidine blue and examined under a light microscope. Àreas of interest were selected and thin sections were cut on an LKB ultratome IV and contrasted with uranyl acetate and lead citrate before being examined in a JEOL 1200 EX electron microscope.

STATISTICS

Means and standard deviations were calculated from individual values by standard procedures. The Mann-Whitney U test was used for comparing cross sectional areas, diameters, and form factors because of non-Gaussian distribution of these variables.

\section{Results}

NORMAL PALATO-PHARYNGEAL MUSCLES

In the muscle biopsies obtained from the control group (fig 1a-b) a normal checkerboard pattern of type I and II fibres was seen under light microscopy when the fibres were stained for myosin ATPase at $\mathrm{pH} 9 \cdot 4$. When the fibres were stained for ATPase at $\mathrm{pH} 4 \cdot 6$, subtypes IIA and IIB were discerened as in normal extremity muscles. No type IIC fibres were observed at $\mathrm{pH} 4 \cdot 3$ (not illustrated).

MUSCLE FIBRE CHANGES IN SPECIMENS FROM APNOEA PATIENTS

Most biopsy specimens showed differentiation between type I and II fibres (fig 1c-d) and type IIA and IIB fibres could be identified. Numerous type IIC fibres were also seen in the specimen from one patient.

In all specimens from patients with obstructive sleep apnoea there was an increased size dispersion with atrophic fibres alternating with normal sized or hypertrophied ones. The 
Figure 2 Cross sections of palatopharyngeal muscle from different patients with obstructive sleep apnoea stained for myosin-ATPase at $\mathrm{pH} 9.4$ (a) and NADH-TR $(b, c)$.

Different sizes of fibres in different fascicles are seen in (a). Note an atrophic fascicle $(x)$. The muscle fibres show type grouping.

Many fibres with rounded clear zones (core

fibres) are seen in (b); one is indicated by a thick arrow. Some fibres have a thin rim of increased stainability adjacent to the clear zone (target fibres). An angulated atrophic fibre is indicated by thin arrows.

$A$ detail from (b) is shown at higher magnification in (c). Note that almost all fibres have irregular stain deposits.

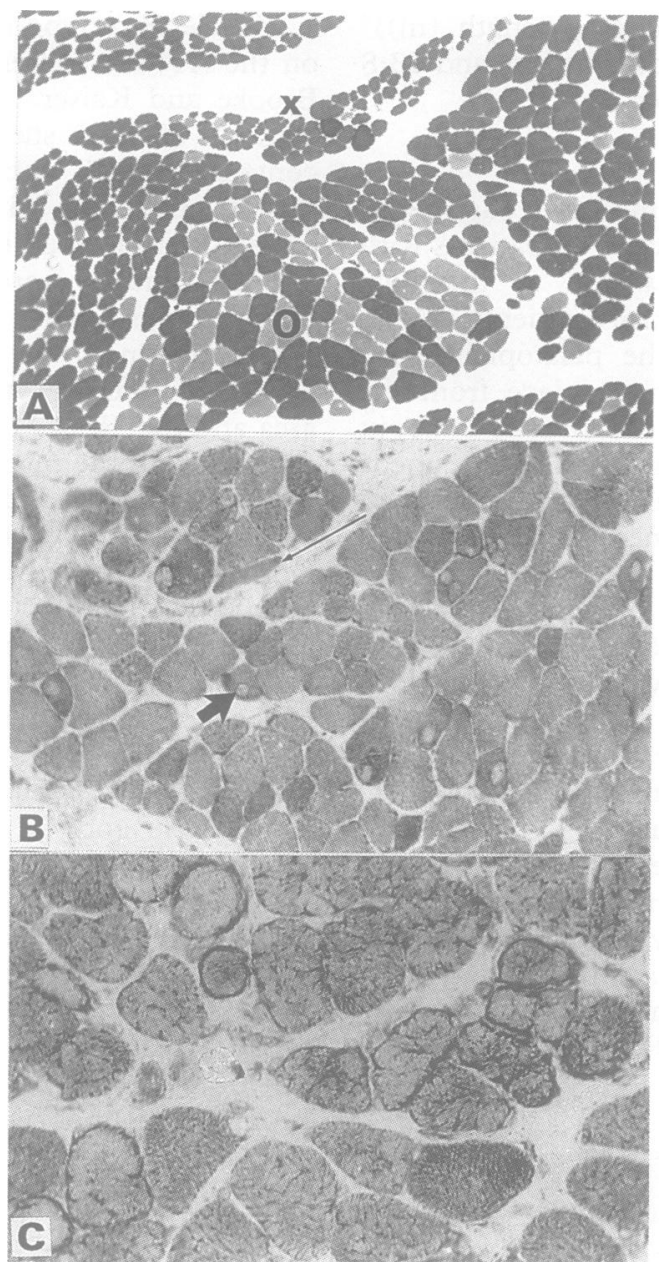

atrophic fibres were rounded or angulated (figs $1 \mathrm{c}-\mathrm{d}$ and $2 \mathrm{~b}$ ). Signs of fascicular atrophy and slight type grouping (fibres of the same type appearing in clusters, fig $2 \mathrm{a}$ ) were also present in all specimens from apnoea patients.

When the fibres were stained for NADH-TR an irregular internal muscle fibre structure was commonly found (figs $1 \mathrm{~d}$ and $2 \mathrm{~b}-\mathrm{c}$ ). "Moth eaten" fibres and fibres with core formations, including the appearance of "target fibres" (fig 2b) regarded as typical of neurogenic muscle
Type 1

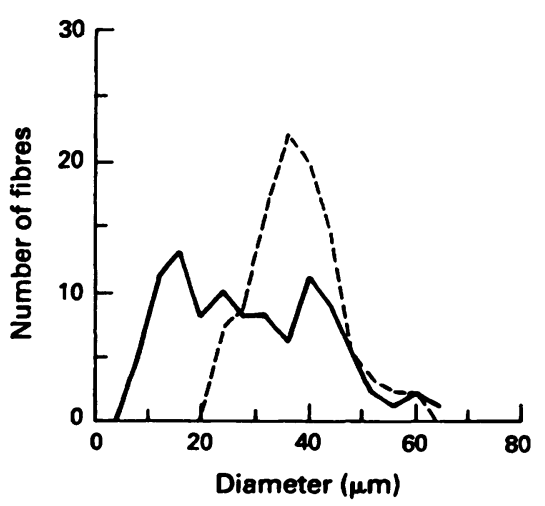

Figure 3 Distribution of calibers (lesser diameters) of type I and type II muscle fibres in a biopsy from a control palatopharyngeal muscle compared to one from a patient with obstructive sleep apnoea. Note the Gaussian distribution of muscle fibre diameters in control muscle and irregular fibre size distribution in apnoea patient's muscle.
Cross sectional area, diameter, and form factor of fibres in palatopharyngeal muscles from patients with obstructive sleep apnoea and controls. Values are means (SD)

\begin{tabular}{lcc}
\hline & $\begin{array}{c}\text { Patients with } \\
\text { obstructive sleep } \\
\text { apnoea }(n=8)\end{array}$ & $\begin{array}{c}\text { Controls } \\
(n=7)\end{array}$ \\
\hline Type I fibres: & & \\
Cross sectional area $\left(\mu \mathrm{m}^{2}\right)$ & $970(370)$ & $1050(350)$ \\
Diameter $(\mu \mathrm{m})$ & $27.7(6 \cdot 1)$ & $29.5(4 \cdot 3)$ \\
Form factor $(\mu \mathrm{m})$ & $0.96(0.01)$ & 0.97 \\
& & $(0.01)$ \\
Type II fibres: & & $1130(480)$ \\
Cross sectional area $\left(\mu \mathrm{m}^{2}\right)$ & $950(260)$ & $30.7(6.3)$ \\
Diameter $(\mu \mathrm{m})$ & $27.0(4 \cdot 9)$ & 0.96 \\
Form factor $(\mu \mathrm{m})$ & $0.95(0.00)$ & $(0.01)$ \\
& &
\end{tabular}

disorders, were also found in all specimens from apnoea patients. In some specimens most fibres showed these changes.

MORPHOMETRICAL OBSERVATIONS

In the controls the muscle fibre size had a Gaussian distribution but in the patients with obstructive sleep apnoea there was a nonGaussian distribution with multiple peaks due to an increased proportion of atrophic and hypertrophic fibres (fig 3 ). When the size of the smallest fibre was compared between different individuals, the fibres in the apnoea patients had a significantly smaller area (70 (SD 40) $\left.\mu \mathrm{m}^{2} ; \mathrm{p}<0.05\right)$ than did fibres in the controls (250 (190) $\left.\mu \mathrm{m}^{2}\right)$. Fibres in the apnoea patients also had a lower form factor $(0.76(0 \cdot 11))$ than did fibres in the controls $(0.90(0.03)$; $\mathrm{p}<0.05$ ), indicating that the atrophic fibres in the apnoea patients were more angulated.

The mean cross sectional area, diameter, and form factor in type I and II fibres did not differ between patients with obstructive sleep apnoea and controls, but these figures may be deceptive due to the difference in fibre size distribution between the two groups as shown in figure 3. The mean values for cross sectional area, diameter and form factor of type I and II fibres in apnoea patients and controls are given in the table.

MUSCLE FIBRE ULSTRASTRUCTURE

A fibre size dispersion was obvious in the cryosections evaluated by TEM. Structural changes corresponding to the irregularities seen under light microscopy were seen in atrophic as well as in normal fibres. In some otherwise normal looking fibres there were enlarged triads. Structurally altered fibres showed myofibrillar loss, increased number of vesicles, and rough $Z$ line material (indicating myofibrillar abnormalities). An increase in the number of ribosomes and polysomes was also discerned, indicating that the muscle fibre degeneration stimulates regenerative effort. Dense bodies, autophagic vacuoles, and lipofucsin bodies were common. Significant mitochondrial abnormalities were seen only in seriously damaged fibres.

\section{Discussion}

The size and stiffness of the upper airway depends on the activity of the paired palatal, 
pterygoid, genioglossal, intrinsic pharyngeal, and hyoid muscles. The separate contribution of each of these muscles is not known and the coordinated action is even more difficult to define. ${ }^{1}$ In this study we examined the palatopharyngeal muscle since it is well defined and easily accessible by tonsillectomy and uvulopalatopharyngoplasty.

In normal subjects the activity of the dilating muscles of the upper airway is coordinated with the activity of the inspiratory muscles. The central regulation of this muscular activity is modulated by chemoreceptors and vagal input $^{89}$ and also by local pressure receptors in the mucosa of the upper airway. ${ }^{1011}$ The activity of the dilating muscles of the upper airway is vitally important in maintaining the patency of the airway during inspiration, and a dysfunction of these muscles may be of pathogenetic importance in obstructive sleep apnoea.

Obstructive sleep apnoea is strongly correlated with obesity and therefore it is not surprising that the patients had a higher body mass index than the controls. However, the pathological structural and ultrastructural changes found in the muscle biopsies from the apnoea patients in this study are not found in obesity. $^{12}$

Data on the normal occurrence and appearance of type I and II fibres in the palatopharyngeal muscle in humans have been lacking. Our findings in the control group indicate that the fibre type profile of this muscle, based on staining for ATPase, is the same as in limb muscles commonly used for muscle biopsy studies. ${ }^{6}$ The occurrence of muscle fibre types and the size relation between type I and II fibres in our control biopsy specimens from the palatopharyngeal muscle were comparable with those of the quadriceps femoris muscle. The mean size of the type I and II fibres, however, was $<25 \%$ of the size of the type I and type II fibres found in quadriceps femoris and many other limb muscles. ${ }^{13}$

In all specimens from the eight apnoea patients the structural, ultrastructural, and histochemical data indicated abnormalities in muscle fibre size, fibre type distribution, and fibre structure. The changes observed were: atrophy with a fascicular distribution and increased number of angulated atrophic fibres; a twin or multiple peak distribution of the fibre size spectrum; and an abnormal distribution of fibre types in many muscle fascicles corresponding to "type grouping." All these changes indicate a neurogenic alteration. ${ }^{6}{ }^{1415-16}$ We found no evidence for a primary myopathy affecting patients with obstructive sleep apnoea.

Stauffer found an increased amount of skeletal muscle and fat in the uvula of apnoea patients as compared to control subjects from an autopsy group. ${ }^{6} \mathrm{He}$ speculated that increased muscular exercise could result in muscular hypertrophy in the pharynx, with increased muscular mass leading to airway obstruction. In this study we did not perform a similar macromorphological analysis of the size of the palatopharyngeal muscle or the tissue composition in the pharynx. Our muscle biopsy findings indicate a neurogenic muscular lesion with atrophy of muscle fibres. We also examined the uvular muscle of the first five patients in the apnoea group. In two the uvular muscle showed complete atrophy and in the others we found the same pathological changes with fibre atrophy as in the palatopharyngeal muscle.

The mean muscle fibre size in our specimens from the palatopharyngeal muscle in patients with obstructive sleep apnoea did not deviate from that in the control group. This is explained by the quantitative data on muscle fibre size showing that hypertrophy of some fibres in the muscles balanced the atrophy of others. It is known that hypertrophy may develop in denervated muscle fibres as a result of passive mechanical stretch. ${ }^{17}$ Such mechanical stretch takes place during each obstructive event in the pharynx of patients with obstructive sleep apnoea. Stretch-induced hypertrophy of muscle cells may be combined with a relative decrease of myofibrillar material and a relative increase of sarcoplasm. ${ }^{18}$ In fact we found many non-atrophic fibres with myofibrillar loss.

The neurogenic myopathy found in the palatopharyngeal muscle in this study may be a primary manifestation in obstructive sleep apnoea or a secondary phenomenon due to the trauma of repetitive and prolonged stretching of the pharyngeal structures during apnoeic episodes. Multiple system atrophy with autonomic failure may cause denervation of the upper airway muscles ${ }^{19}$ but it is a rare condition not occurring with increased incidence in obstructive sleep apnoea, where there seems to be a sympathetic overreactivity. ${ }^{20}$ A disturbance of motor regulation and also of the muscle fibre properties in the dilating muscles of the upper airway may be important in the mechanisms which cause the abnormal airway collapse seen in patients with obstructive sleep apnoea.

This study was supported by grants from the Swedish Society of Medicine, the Swedish Medical Research Council (project 3875 and 8651), the Torsten and Ragnar Söderberg foundation, and the Karolinska Institute. We are indebted to Ms Birgitta Hedberg, Ms Birgitta Lindegren, and Mrs Lillebil Stuart for skillful technical assistance. The authors thank Drs Brit Nordlander and Eva Svanborg for valuable advice, the latter also for performing the sleep apnoea analyses.

1 Remmers JE. Anatomy and physiology of upper airway obstruction. In: Kryger M, Roth T, Dement W, eds. Principles and practice of sleep medicine. Philadelphia: Saunders, 1989:525-36

2 Stauffer JL, Buick MK, Bixler EO, Sharkey FE, Abt AB, Manders EK, et al. Morphology of the uvula in obstrucManders EK, et al. Morphology of the uvula in obstruc-

tive sleep apnoea. Am Rev Respir Dis 1989;140:724-8.
Fujita S. UPPP for sleep apnea and snoring. Ear Nose Throat 1984;63:227-35.

4 Alihanka J, Vaahtoranta K, Saarikivi I. A new method for long-term monitoring of the ballistocardiogram, hear rate and respiration. Am $\mathcal{F}$ Physiol 1981;240:R384-92.

5 Svanborg E, Larsson H, Nordlander B, Pirskanen R. A limited diagnostic investigation for obstructive sleep apnea syndrome: oximetery and static change sensitive bed. Chest 1990;98:1341-5.

6 Dubowitz V. Muscle biopsy-a practical approach. 2nd ed. London: Baillière Tindall, 1985.

7 Brooke MH, Kaiser KK. Muscle fibre type: How many and what kind? Arch Neurol 1970;23:369-79.

8 Önal E, Lopata M, O'Connor TD. Diaphragmatic and geniglossal electromyogram response to $\mathrm{CO}$ rebreathin in humans. F Appl Physiol 1981;50:1052-5.

9 Weiner D, Mitra J, Salamone J, Cherniack NS. Effect of chemical stimuli on nerves supplying upper airway muscles. I Appl Physiol 1983;52:530-6. 
10 Mathew OP. Upper airway negative pressure effects on respiratory activity of upper airway muscles. $\mathcal{f} A$ ppl Physiol 1984;56:500-5.

11 van Lunteren E, Van de Graaff WB, Parker DM, Haxhiu MA, Strohl KP, Cherniack NS. Nasal and laryngeal reflex responses to negative upper airway pressure. $₹ \mathrm{Appl}$ responses to negative
Physiol 1984;56:746-52.

12 Wadström C, Larsson L, Knutsson E, Edström L. The effect of excessive weight loss on skeletal muscle in man European fournal of Surgery 1991;157:347-54.

13 Edström L, Nyström B. Histochemical types and size of fibres in normal human muscles. Acta Neurol Scand 1969;45:257-69.

14 Edström L, Kugelberg E. Histochemical mapping of motor units in experimentally reinnervated rat muscle. Experen tia $1969 ; 25: 1044-6$.

15 Kugelberg E, Edström L, Abbruzzese M. Mapping of motor units in experimentally reinnervated rat muscle. $\mathcal{F}$ Neurol
Neurosurg Psychiatry 1970;33:319-30.

16 Morris CJ, Raybould JA. Fibre type grouping and end-plate diameter in human skeletal muscle. $f$ Neurol $S c i$ 1971;13:181-7.

17 Sola OM, Chistensen DL, Martin AW. Hypertrophy and hyperplasia of adult chicken anterior latissimus dorsi muscles following stretch with and without denervation. Exp Neurol 1973;41:76-80.

18 Gutmann E, Hajek I. Differential reaction of muscle to excessive use in compensatory hypertrophy and increased phasic activity. Physiol Bohemslov 1971;20:205-12.

19 Guindi GM, Bannister R, Gibson WPR, Payne JK. Laryngeal EMG in multiple system atrophy with autonomic failure. $\mathcal{f}$ Neurol Neurosurg Psychiatry 1981;44:49-53.

20 Svanborg E, Carlsson-Nordlander B, Larsson H, Sachs C, Kaiser $\mathrm{L}$. Autonomic nervous system function in patients with primary obstructive sleep apnea syndrome. Clinical Autonomic Research 1991;1:125-30.
Early accounts of dystophia myotonica In 1886 Erb reported his findings of electrical reactions and histology of a number of families suffering from Thomsen's myotonia congenita.' He remarked on "genetic heterogeneity" but this embraced cases with muscle wasting who may have been examples of dystrophia myotonica, as were later cases reported as "myotonia congenita with muscle atrophy". Hoffmann" reported a 35 year old man with myotonia and wasting of the facial, sternomastoid and forearm muscles. In 1900 he observed a brother and sister with facial, sternomastoid and distal limb wasting, weakness and myotonia. Other convincing examples were described by Rossolimo, Nonne, Pässler, and Curschmann. ${ }^{3}$ Of less certain nosology is a case claimed by some as the first by Danna. ${ }^{4}$ A man developed from the age of 20 myotonia, ptosis, impotence and mental symptoms; he had no recognised facial weakness but a supinator harpoon biopsy showed internal nuclei in the myofibrils.

Steinert described nine patients, three to the Leipzig Medical Society in 1904, six (including two brothers) in his paper. ${ }^{5} \mathrm{He}$ was mistaken in attributing the muscle atrophy he had noticed to myotonia. In addition to the characteristic ptosis, facial and sternomastoid weakness he remarked on the weak voice, areflexia and in four patients-testicular atrophy. His one necropsy case confirmed the atrophy of muscle spindles and normal nerve fibres within muscles; he found dorsal column atrophy in the cord-probably due to coincidental tabes.

Batten and Gibb recognised the disease as an entity distinct from myotonia congenita and their 1909 paper $^{6}$ includes the first photograph unmistakeably portraying the disease. It is curious that the association with cataracts was not noted till Greenfield's account. ${ }^{7}$ J M S PEARCE

1 Erb W. Die Thomsen'sche Krankheit (Myotonia congenita) Leipzif. cited by Harper PS. In: Myotonic dystrophy, 2nd Leipzif. cited by Harper PS. In:

2 Hoffmann J. Ein Fall von Thomsen'scher Krankheit, complizirt

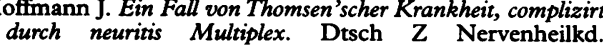
durch neuritis
$1896 ; 9: 272-8$.

3 Curschmann H. Demonstration eines Falles Thomsen'scher Krankheit it ausgedehnten muskelatrophien. Munsch Med Wochenschrift 1906;53:1281-2.

4 Dana CL. An atypical case of Thomsen's disease (myotonia congenita). Medical record, NY 1988;33:433-5.

5 Steinert $\mathrm{H}$. Ein neuer Fall von atrophischer Myotonie: ein Nachtag zu meiner Arbeit in Bild 37. Dtsch $Z$ Nervenheilkd 1910;39:168-73.

6 Batten FE, Gibb HP. Myotonia atrophica. Brain 1909;32:187-205.

7 Greenfield JG. Notes on a family of "myotonia atrophica" and early cataract with a report on an additional case of myotonia atrophica. Rev Neur Psych (Edin) 1911;9:169-81. 